\title{
Pinocembrin mitigates depressive-like behaviors induced by chronic unpredictable mild stress through ameliorating neuroinflammation and apoptosis
}

Wei Wang ${ }^{1 *+} \mathbb{D}$, Lili Zheng ${ }^{2+}$, Lijun $X u^{1}$, Jianglong Tu${ }^{1}$ and Xunhu Gu ${ }^{1}$

\begin{abstract}
Background: The majority of patients with chronic fatigue have a risk of comorbidity with depression. Pinocembrin (PB) is a kind of flavonoid molecule isolated from honey and propolis and has antimicrobial, anti-inflammatory, antioxidant, and anticancer function. The purpose of the current study was to determine the possible function of PB on treatment of depression.

Methods: A chronic unpredictable mild stress (CUMS) mouse model was established to mimic the depressive-like behaviors in vivo. The depressive-like behaviors of CUMS mice were measured by sucrose preference test (SPT), open field test (OFT), forced swim test (FST) and tail suspension test (TST). The concentration of reactive oxygen species (ROS), malondialdehyde (MDA) and the activity or superoxide dismutase (SOD) were detected by commercial kit. The inflammatory factor including interleukin (IL)-1 $\beta$, tumor necrosis factor (TNF)- $\alpha$, IL-10 and transforming growth factor (TGF)- $\beta$ were examined.

Results: We found that PB alleviated the decreasing of sucrose preference and body weight. CUMS mice significantly increased the immobility time but decreased latency to abandon in FST, increased the immobility time in TST, and reduced crossing score and rearing score in OFT, whereas these changes were reversed by PB treatment. More importantly, PB decreased the concentration of ROS and MDA, but increased the SOD activity, suggesting that it could protected against oxidative stress in CUMS mice. Interestingly, PB inhibited cell apoptosis and regulated inflammatory factors expression in hippocampus of CUMS mice. Moreover, PB activated Nrf2/HO-1 signal pathway but inhibited the phosphorylation of NF-kB.
\end{abstract}

Conclusions: In conclusion, PB mitigated CUMS-induced depressive-like behaviors through ameliorating neuroinflammation and apoptosis.

Trial registration: Not Applicable.

Keywords: Pinocembrin, Chronic unpredictable mild stress, Neuroinflammation, Apoptosis, Oxidative stress

\footnotetext{
* Correspondence: TRFD7ui2@163.com

'Wei Wang and Lili Zheng contributed equally to this work.

'Department of Neurology, The Second Affiliated Hospital of Nanchang

University, No.1 Minde Road, Nanchang City, Jiangxi Province, China

Full list of author information is available at the end of the article
}

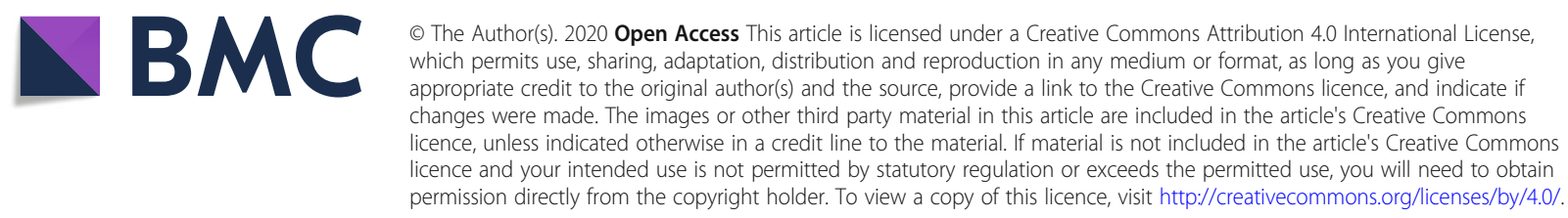




\section{Background}

Depression is a severe and recurrent disease, which is characterized by persistent depressed mood and impaired cognitive functions, even leads to suicide or selfharm (Coleman et al. 2019; Butter et al. 2019). More patients have been affected in the world, thus it becomes a serious personal pain and social problem. Currently, more than 20 different antidepressants are used to treat depression, however, there is still a large of patients suffering the pains which are brought from depression (Kessler and Bromet 2013). The main reason of the poor effect of antidepressant treatment is the ambiguity of the pathophysiology of depression and the molecular mechanism of antidepressant treatment (Riddle et al. 2017; Peng 2018). Therefore, further studies about the pathogenesis of depression and therapeutic strategies are needed.

The brain is susceptible to oxidative stress because of its high iron content, high oxygen consumption, low antioxidant capacity and peroxidation of fatty acids (Madrigal et al. 2006; Salim 2017). Therefore, cerebral oxidative stress is an important mechanism of brain dysfunction, especially depression and anxiety (Tell and Gustincich 2009). In a previous study, cell apoptosis is considered as a mechanism for promoting stress-related mood disorders (Lee et al. 2014). Cell death occurs in specific groups of neurons, which are caused by chronic stress. In the circumstances, antidepressants have been shown to improve repetitive stress-induced cognitive impairment (Kwon et al. 2013). In clinical patients, the release of pro-inflammatory cytokines, especially interleukin-1 cytokines (IL-1) and tumor necrosis factor (TNF-), is higher in depressed patients compared with normal patients, indicating that inflammation plays an vital role in the pathophysiology of depression (AlHakeim et al. 2015; Eyre et al. 2016; Hannestad et al. 2011). In addition, antidepressant treatment reduces serum inflammatory cytokines (Yamawaki et al. 2018). Higher expression of pro-inflammatory cytokines have been identified in depressed animal models (Jiang et al. 2017a). Therefore, these findings suggest that the antiinflammatory and anti-apoptotic functions play vital roles in depression treatment.

Natural products are valuable and novel resources for drug development, such as propolis. Pinocembrin (5,7dihydroxyflavanone, molecular formular: $\mathrm{C}_{15} \mathrm{H}_{12} \mathrm{O}_{4}$, molecular weight: $256.25 \mathrm{~g} / \mathrm{mol}$ ), is a kind of flavonoid, which is isolated from propolis and honey (Rasul et al. 2013). The PB showed antioxidant and antiinflammatory abilities and neuroprotective functions in vivo and in vitro (Reddy et al. 2013; Lan et al. 2016). PB alleviates inflammation, oxidative interference, and apoptosis in the hippocampus of the brain ischemiareperfusion rat model (Saad et al. 2015). However, it has not been reported whether it can alleviate depressionlike behaviors with the mechanism of inflammation and apoptosis. The purpose of our study was to study the regulation of $\mathrm{PB}$ on depression in a CUMS-induced depression mouse model.

\section{Methods}

This study was obeyed the Guide for the Care and Use of Laboratory Animals and the protocol was approved by the Ethics Committee of The Second Affiliated Hospital of Nanchang University.

\section{Animals and treatment}

Total of 40 male C57BL/6 J mice (six-week-old) were purchased from Huafukang Company. Every mouse was fed adaptively under a normal $12 \mathrm{~h}$ light/dark cycle at $23 \pm 2{ }^{\circ} \mathrm{C}$ (humidity $45 \%-55 \%$ ) for 2 weeks before experiments began. During the study, the mice were given food and water every day. The mice were randomly divided into five groups ( $n=8$ per group):

(a) Control;

(b) Control+ $10 \mathrm{mg} / \mathrm{kg} \mathrm{PB}$;

(c) Chronic unpredictable mild stress (CUMS);

(d) CUMS+ $10 \mathrm{mg} / \mathrm{kg} \mathrm{PB}$;

(e) CUMS $+10 \mathrm{~mL} / \mathrm{kg}$ imipramine hydrochloride (IMI);

The CUMS experiments were performed for 6 weeks. At the 4th week, the PB was administrated once a day for 3 weeks by oral gavage. The IMI treatment was served as a positive control, the IMI were administrated every day during the UCSM experiments by oral gavage. The UCSM and SPT, depressive-like behavioral assessment were carried out in the order presented in Fig. 1A.

\section{Chronic unpredictable mild stress (CUMS)}

To explore the effects of PB on depression-like behaviors in animals, the CUMS-induced mouse model was used in this study. The control group did not receive any of the chronic unpredictable mild stresses experiment, whereas CUMS-induced mice received single housed and subjected to one of the following stressors once every day in a randomized fashion: (I) lake of food for $24 \mathrm{~h}$, (II) lake of water for $24 \mathrm{~h}$ (III) lighting for $24 \mathrm{~h}$, (IV) cage tilt $\left(45^{\circ}\right),(\mathrm{V})$ the damp environment (per $100 \mathrm{~g}$ padded add $200 \mathrm{ml}$ of water), (VI) exposed to the external environment, (VII) the light light/dark cycle, (VIII) alternately suspension for $10 \mathrm{~min}$, (IX) exposed to an empty bottle, $(\mathrm{X})$ clip tail $(1 \mathrm{~min}, 1 \mathrm{~cm}$ in from the tail to start), (XI) oscillation for $5 \mathrm{~min}$, (XII) white noise. Mice were subjected to different stressors daily for 42 days in an unpredictable way. Finally, the mice were sacrificed at the 43th day. The mice anesthetized with sodium pentobarbital $(100 \mathrm{mg} / \mathrm{kg})$ and fentanyl $(0.05 \mathrm{mg} / \mathrm{kg})$. The 


\section{A}

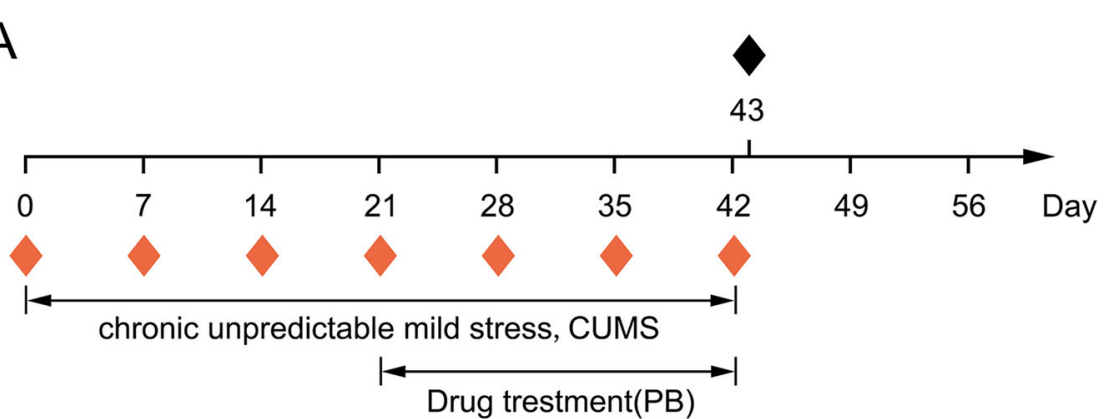

\section{: SPT and weight test \\ Depressive-like behavioral assessment}

B

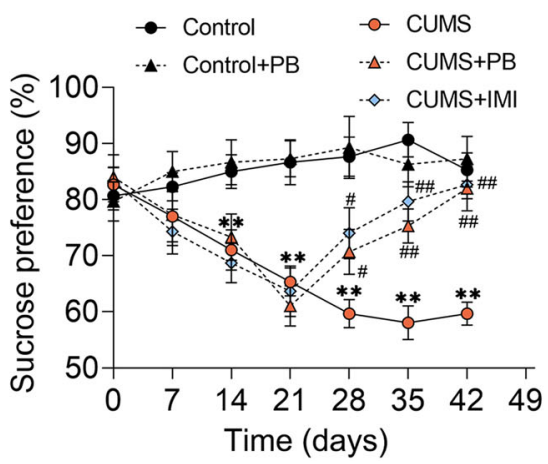

C

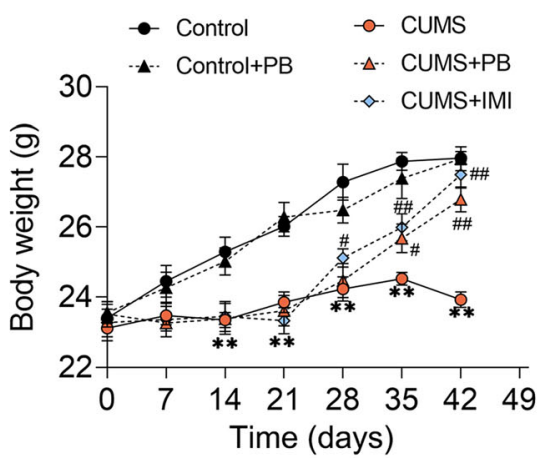

Fig. 1 PB alleviated sucrose preference and body weight in CUMS mice (A) Experimental schedule. A total of 40 C57BL/6 J mice randomly divided into 5 groups: (a) Control; (b) Control+PB; (c) CUMS; (d) CUMS+PB; (e) CUMS+IMI. Meanwhile, the IMI treatment was served as a positive control, and IMI was administrated every day during the UCSM experiments by oral gavage. The unpredictable chronic mild stress (UCMS) protocols and PB treatment lasted 6 weeks and 3 weeks, respectively. Before the UCMS procedure, the sucrose preference and body weight were measured and lasted for once a week. Sucrose preference was measured in UCMS weekly. The depressive-like behavioral assessment was assessed at the end of the experiments. The sucrose preference (B) and body weight $(\mathbf{C})$ were detected once a week. Eight mice per group. ${ }^{* *} p<0.01$ vs. Control. \#p $<0.05$, \#\#p $<0.01$ vs. CUMS

brains were quickly removed, washed with phosphatic buffer solution (PBS), and post-fixed in $4 \%$ paraformaldehyde overnight at $4{ }^{\circ} \mathrm{C}$ for further experiments.

\section{Sucrose preference test (SPT)}

Anhedonia is the inability to experience pleasure from rewarding or enjoyable activities, which is a core symptom of depression in humans, and can be assessed by SPT assay (Liu et al. 2018). In this experiment, weight was weighed every 7 days for 6 weeks. In addition, SPT was administered every 7 days before and after induction in CUMS. First, the mice were placed in separate cages, each adapted to a $1 \%$ sucrose solution (w/v). After $24 \mathrm{~h}$ of exposure to two bottles of sucrose solution, a bottle of $1 \%$ sucrose solution in each cage was replaced with a bottle of water and exposed for another $24 \mathrm{~h}$. Then, all mice were deprived of water and food for $24 \mathrm{~h}$, and SPT was administered for $12 \mathrm{~h}$. During this time, the mice were free to use two bottles, one contained a $1 \%$ sucrose solution and the other was only water. The location of the bottle was changed during the 6-h test to avoid possible side effects. The sucrose preference was calculated as follows: SPT $(\%)=(($ sucrose intake $(\mathrm{g}) /$ (sucrose intake $(\mathrm{g})+$ water intake $(\mathrm{g}))) \times 100$.

\section{Forced swim test (FST)}

The FST is a model of depressive-like behavior (Yankelevitch-Yahav et al. 2015). To allow adaptation to the environment, all mice were taken by a $6 \mathrm{~min}$ FST. The mice in each group were placed in a water container 25 $\mathrm{cm}$ high and $10 \mathrm{~cm}$ in diameter $\left(10 \mathrm{~cm}\right.$ deep; $\left.23 \pm 2{ }^{\circ} \mathrm{C}\right)$. The total 6-min process included the first 2-min unmeasured swim and the subsequent 4-min swim. The immobility time of $4 \mathrm{~min}$ after swimming was recorded by the software tracking analysis system (Xinsoft Information Technology, China). The latency to abandon is thought to be the time which ranged from the time that 
the animal was introduced into the pool to the time it first stopped completely (at least $2 \mathrm{~s}$ ).

\section{Tail suspension test (TST)}

The TST assay is a widely used models for assessing antidepressant-like activity in animal models (Cryan et al. 2005). In this study, the TST assay was performed as a previous reference described (Jiang et al. 2017b). Each mouse was hung $50 \mathrm{~cm}$ away from the ground for $6 \mathrm{~min}$, with its tail attached to the hook (about $2 \mathrm{~cm}$ from the end). Calculate the immobility time for the last $4 \mathrm{~min}$. The mice are considered to be motionless only when they are completely motionless.

\section{Open field test (OFT)}

The OFT experiment was a test of anxiety-related behavior and locomotion and performed as described previously (Wu et al. 2019; Belzung and Griebel 2001). In short, the instrument is divided into 12 equal squares. The rats were then placed in the middle of the field and allowed to explore their surroundings Finally, the numbers of square crossings, rearing and grooming scores within 5 min of free activity were recorded.

\section{Measurement of MDA and SOD}

The hippocampus from mice were homogenized $(10 \%$ $\mathrm{w} / \mathrm{v})$ with PBS and centrifuged for collecting supernatants. The levels of MDA and the activity of SOD in the supernatant were respectively measured by MDA Assay Kit (Abnova, USA) and SOD Assay Kit (Abnova, USA), according to the manufacturer's instructions.

\section{Determination of ROS level}

The levels of ROS in hippocampus were evaluated by using 2, 7-dichlorofluorescindiacetate (DCFDA) Kit (Sigma, China). Briefly, hippocampus homogenate was washed with PBS, mixed with DCFDA (dissolved with methanol) and then incubated in water for $15 \mathrm{~min}$ at $37^{\circ} \mathrm{C}$. After that, the samples were stained by DAPI. ROS images were measured using a DCFH-DA fluorescence probe under a fluorescence microscope with a magnification of $400 \times$. Fluorescence intensity was calculated using a fluorometer at $488 \mathrm{~nm}$ excitation wavelength and $525 \mathrm{~nm}$ emission wavelength (Hemmati et al. 2018).

\section{Terminal-deoxynucleoitidyl Transferase mediated Nick}

\section{end labeling (TUNEL) assay}

The cell apoptosis was detected by FragEL ${ }^{\mathrm{Tm}}$ DNA Fragmentation Dectection Kit (Merck-Calbiochem, USA). The hippocampus from mice were embedded in paraffin, and cut into $5-\mu \mathrm{m}$ slices. After deparaffinization, the tissue sections were incubated with proteinase $\mathrm{K}$ for 25 min and incubated with $\mathrm{H}_{2} \mathrm{O}_{2}$ in methanol $(8 \mathrm{~min})$.
Next, the sections were placed in the TUNEL reaction mixture and incubated in darkness for $60 \mathrm{~min}$. After washing by $\mathrm{PBS}$, the sections were incubated with streptavidin-horseradish peroxidase (HRP) conjugate, and $\mathrm{DAB}$ was served as chromogen substrate. The nuclear staining of cell in intense and brown was TUNEL-positive.

\section{Immunohistochemistry assay}

The hippocampus from mice after deparaffinization were incubated in citrate buffer solution, and then were blocked with $10 \%$ goat serum. After that, slides incubated with the Anti-Cleaved Caspase- 3 antibody (dilution:1:300; Abcam, USA) at $4{ }^{\circ} \mathrm{C}$ overnight. Then, the sections were stained with DAB chromogen substrate in the dark. Finally, the images were recorded by a microscope.

\section{Measurement of TNF- $\alpha$, IL-1 $\beta$, TGF- $\beta$ and IL-10}

The concentrations of TNF- $\alpha$, IL-1 $\beta$, TGF- $\beta$ and IL-10 in hippocampus from mice were measured by Mouse IL-1 $\beta$ ELISA Kits (MULTI SCIENCES, China), Mouse TNF- $\alpha$ ELISA Kits (MULTI SCIENCES, China), Mouse IL-10 ELISA Kits (MULTI SCIENCES, China) or Mouse TGF- $\beta$ ELISA Kits (MULTI SCIENCES, China).

\section{Quantitative real time polymerase chain reaction (qRT- PCR)}

Total RNA was extracted from hippocampus in mice using TRIzol reagent (Takara, Japan). And then the cDNA was synthesized by Prime-Script ${ }^{\mathrm{m}} \mathrm{RT}$ regent Kits (Takara, Japan). The mRNA expression levels were measured using qRT-PCR by SYBR Premix Ex Taq (Takara, Japan). The critical threshold cycle $(\mathrm{Ct})$ value was determined and the relative quantification data were calculated with the $2^{-\Delta \Delta \mathrm{Ct}}$ method, the GAPDH was served as a reference. The primers were as follows: IL-1 $\beta$-forward: 5 '-GAGTCTGCACAGTTCCCCAA-3', IL-1 $\beta$-reverse: $5^{\prime}$-TGTCCCGACCATTGCTGTTT-3'; TNF$\alpha$-forward: 5'-CGTC AGCCGATTTGCCATTT-3', TNF- $\alpha$ reverse: 5 '-CTCCCTCAGGGGTGTCCTTA-3'; TGF- $\beta$ forward: 5'-GAACTCGCTTTGTCTCCA-3', TGF- $\beta$-reverse: 5'-TACAGTCGCAGTATAACCTCA-3'; IL-10-forward: 5' TCTCCGAGATGCCTTCAGCAGA-3', IL-10-reverse: 5' TCAGACAAGGCTTGGCAACCCA-3'; GAPDH-forward: 5'-ATGGGGAAGGTGAAGGT-3', GAPDH-reverse: 5' AAGCTTCCCGTTCTCAG-3'.

\section{Western blot}

The protein from the tissues of mice were lysed with RIPA lysis buffer (Beyotime, China). Then, the protein concentrations were detected by a $\mathrm{BCA}$ protein kit (Beyotime, China). Next, equal proteins from different groups were separated by SDS-poluacrylamide gel electrophoresis, transferred onto a PVDF membrane. After 
washed by TBST buffer for three times, the membrane was incubated with first antibodies: Nrf2 antibody (dilution: 1:400; Abcam, USA), HO-1 antibody (dilution: 1:400; Abcam, USA), Bcl-2 antibody (dilution: 1:800; Proteintech, USA), Bax antibody (dilution: 1:800; Proteintech, USA), pNF-kB antibody (dilution: 1:400; Abcam, USA), NF-kB antibody (dilution: 1:400; Abcam, USA), GAPDH antibody (dilution: 1:10000; Proteintech, USA) overnight at $4{ }^{\circ} \mathrm{C}$, followed by incubation with the appropriate secondary antibodies (dilution: 1:5000; Beyotime, China) for $2 \mathrm{~h}$. The GAPDH was served as a loading control.

\section{Data analysis}

All Data were expressed as mean \pm standard deviation (SD) and analyzed with were analyzed by one-way analysis of variance (ANOVA) or two-way ANOVA by GraphPad Prism 7.0. $P<0.05$ was considered significant.

\section{Results}

\section{PB alleviated sucrose preference and body weight in} CUMS mice

The UCSM and SPT, depressive-like behavioral assessment were presented in Fig. 1A. Firstly, the CUMS treatment markedly reduced the sucrose preference and body weight than control mice. Nevertheless, the PB or IMI treatment could alleviate the decreasing of sucrose preference and body weight (Fig. 1B and C). Thus, we could conclude that PB alleviated sucrose preference and body weight in CUMS mice.

\section{PB alleviated depressive-like behaviors of CUMS mice}

To clarify the function of $\mathrm{PB}$ on CUMS mice, the depressive-like behaviors was detected by Depressivelike behavioral assessment. Six-week CUMS experiments markedly increased the immobility time but decreased latency to abandon in FST compared to control mice. Treatment with PB or IMI significantly decreased immobility time but increased latency to abandon in FST compared to that of the CUMS (Fig. 2a). Moreover, compared to control mice, $\mathrm{PB}$ observably increased the immobility time in TST, as well as IMI (Fig. 2b). The PB or IMI treatment significantly increased crossing score and rearing score compared to CUMS mice in OFT. Nevertheless, there was no significant difference among the five groups in grooming score (Fig. 2c). These

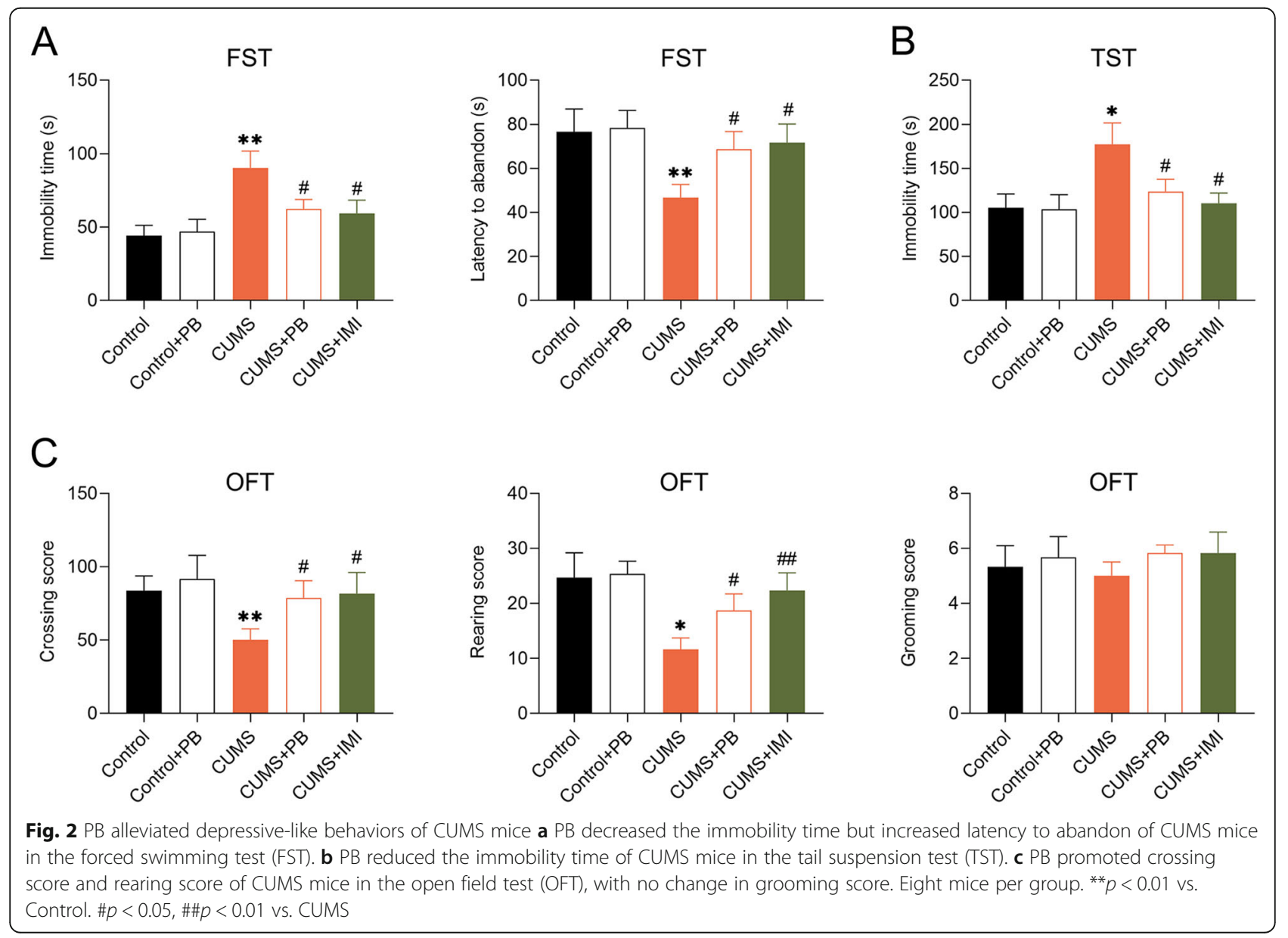


findings indicated that $\mathrm{PB}$ alleviated depressive-like behaviors of CUMS mice.

\section{PB protected against oxidative stress in hippocampus of CUMS mice}

To further investigate the role of $\mathrm{PB}$ in the oxidative stress, the levels of ROS, MDA and SOD in the hippocampus of CUMS-treated mice were measured. ROS level was significantly promoted in the hippocampus of CUMS mice compared to control mice. PB or IMI treatment markedly inhibited the ROS production (Fig. 3A and B). The concentration of MDA (Fig. 3c) was significantly increased but the activity of SOD (Fig. 3d) were markedly decreased in CUMS-induced mice. Nevertheless, treatment with PB or IMI decreased the concentration of MDA but increased the SOD activity in CUMSinduced mice, indicating that $\mathrm{PB}$ protected against CUMS-induced hippocampal oxidative stress.

\section{PB inhibited cell apoptosis in hippocampus from CUMS mice}

The apoptotic neurons in hippocampus was recognized by TUNEL staining and IHC assay in the hippocampus from mice. In the Control and Control+PB mice, TUNEL-positive cells or cleaved caspase-3-positive cells were rare or absent in hippocampus (Fig. 4a). Nevertheless, the TUNEL-positive cells or cleaved caspase-3positive cells were present in hippocampus from CUMS mice. Interestingly, after the $\mathrm{PB}$ or IMI treatment, the TUNEL-positive cells or cleaved caspase-3-positive cells were redyced in hippocampus from CUMS mice (Fig. $4 b)$. These findings proved that $\mathrm{PB}$ inhibited the cell apoptosis in hippocampus from CUMS mice.

\section{PB regulated inflammatory factors expression in CUMS mice}

Compared to control mice, the mRNA levels of TNF- $\alpha$ and IL-1 $\beta$ were significantly increased, but the mRNA levels of TGF- $\beta$ and IL-10 were markedly decreased in CUMS mice. Moreover, the treatment of $\mathrm{PB}$ or IMI could reduce the expression of TNF- $\alpha$ and IL- $1 \beta$ but increase the expression of TGF- $\beta$ and IL-10 (Fig. 5a-d). Interestingly, the trends of TNF- $\alpha$, IL- $1 \beta$, TGF- $\beta$ and IL10 concentrations in hippocampus of mice, which was determined by ELASA assay, were similar to the expression of them (Fig. 5e-h). These findings strongly proved that $\mathrm{PB}$ regulated inflammatory factors expression in CUMS mice.

\section{PB activated Nrf2/HO-1 signal pathway but inhibited the phosphorylation of NF-kB}

To further explore how PB ameliorated neuroinflammation and apoptosis, we observed the dynamic changes of Nrf2, HO-1 and p-NF-kB protein levels. From Fig. 6, the expression of $\mathrm{HO}-1$ and $\mathrm{Nrf} 2$ were increased in CUMS mice. Nevertheless, the PB or IMI treatment could induce the Nrf2 and HO-1 expression, indicating that PB alleviated depressive-like behaviors in mice via activating Nrf2/HO-1 signal pathway. Compared to control mice, the $\mathrm{Bcl}-2$ was decreased but the Bax was increased in CUMS mice, whereas these changes were reversed by $\mathrm{PB}$ or IMI treatment, suggesting that PB could inhibited the CUMS-induced cell apoptosis. More importantly, the phosphorylation of NF-kB was observed to promoted in CUMS mice but inhibited by PB or IMI treatment, indicating that $\mathrm{PB}$ alleviated depressive-like behaviors in mice via inhibiting the phosphorylation of NF-kB.

\section{Discussion}

We established a CUMS mouse model to mimic the depressive-like behaviors in vivo. PB alleviated the decreasing of sucrose preference and body weight. CUMS mice significantly increased the immobility time but decreased latency to abandon in FST, increased the immobility time in TST, and reduced crossing score and rearing score in OFT, whereas these changes were reversed by $\mathrm{PB}$ treatment. More importantly, $\mathrm{PB}$ decreased the concentration of ROS and MDA, but increased the SOD activity, suggesting that it could protected against oxidative stress in CUMS mice. Interestingly, PB inhibited cell apoptosis and regulated inflammatory factors expression in hippocampus of CUMS mice. Moreover, $\mathrm{PB}$ activated $\mathrm{Nrf} 2 / \mathrm{HO}-1$ signal pathway but inhibited the phosphorylation of NF-kB. Thus, PB activated Nrf2/ HO-1 signal pathway but inhibited the phosphorylation of NF-kB, and then ameliorated neuroinflammation and oxidative stress, further inhibited cell apoptosis and mitigated CUMS-induced depressive-like behaviors (Fig. 7).

Depression caused mood and anxiety disorders and adversely affect individual's productive life. Currently, natural products are considered good choices to treat $87 \%$ of all categorized human diseases, because of their anticancer, antibacterial, antiparasitic, anticoagulant, and immunosuppressant functions, such as PB (Newman et al. 2003). It has been reported that PB attenuated autonomic dysfunction and atrial fibrillation susceptibility (Ye et al. 2019). Gao J et.al have been pointed that PB inhibits the proliferation and migration but promotes the apoptosis in ovarian cancer cells (Gao et al. 2019). Moreover, PB has been proved to protect hemorrhagic brain primarily through inhibiting toll-like receptor 4 and decreasing M1 phenotype microglia (Lan et al. 2017). These results provided a novel strategy of PB for treating depression. In the present study, we have established a CUMS-induced depression-like behavior mouse model for in vivo study. Interestingly, we demonstrated that $\mathrm{PB}$ alleviated the decreasing of sucrose preference and body weight, and alleviated depressive-like behaviors 


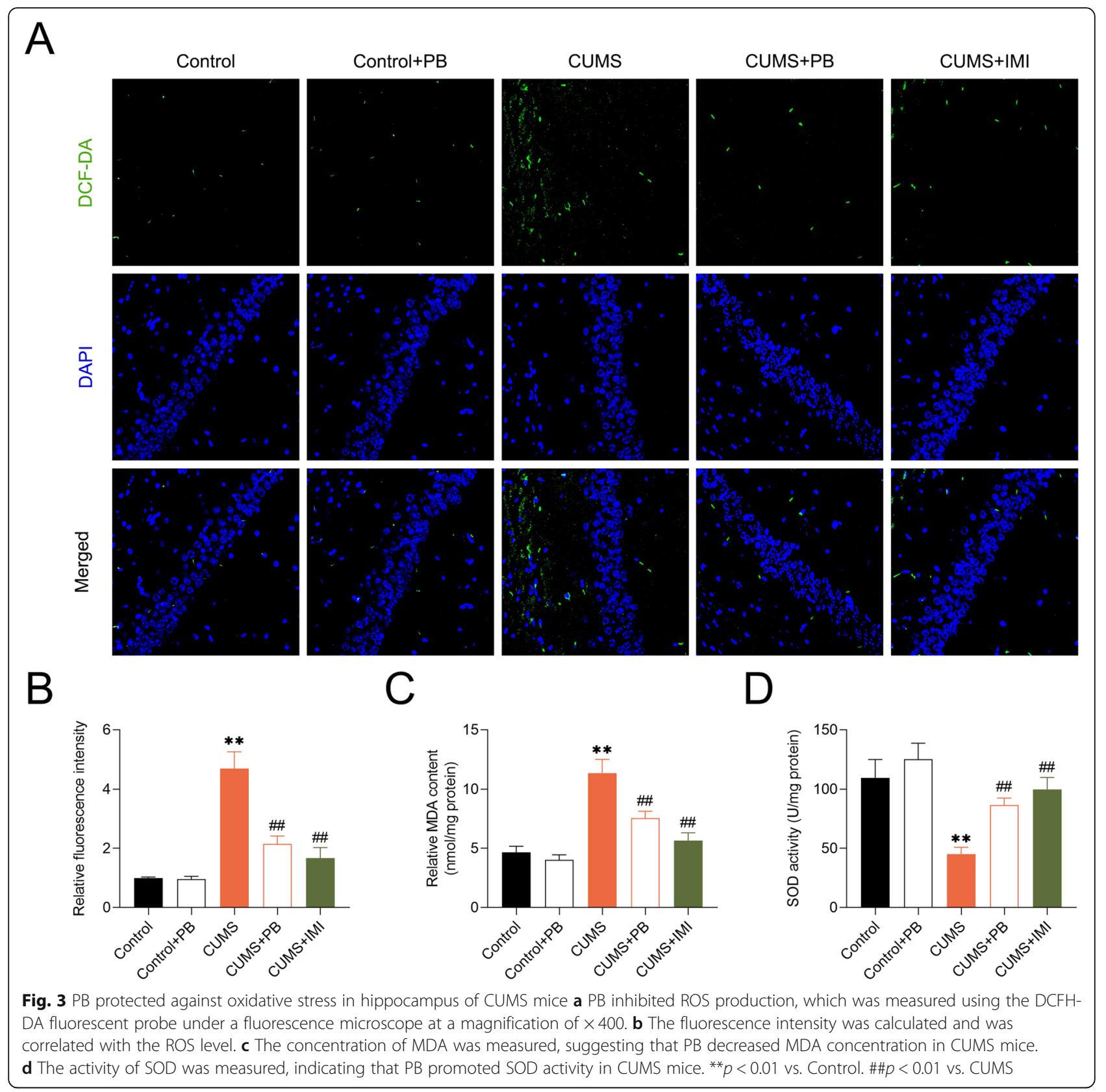

in CUMS mice, indicating that $\mathrm{PB}$ may be a promising novel drug therapeutic for depression. However, the mechanism of how PB affects the depressive-like behaviors in CUMS-induced mice is still unclear.

Postmortem analysis show that the size of neurons is reduced and the apoptosis of neurons is increased in patients with depression (Stockmeier et al. 2004; McKernan et al. 2009). Stress induces cell apoptosis in the hippocampus and reduces neurogenesis in vivo (Kubera et al. 2011). In this study, compared to control mice, we have demonstrated that $\mathrm{PB}$ inhibited cell apoptosis in hippocampus of CUMS-induced mice. Oxidative stress causes mitochondrial membrane depolarization, leading to the release of cytochrome $\mathrm{C}$ from mitochondria into the cytoplasm, which activates the caspase pathway and leads to cell apoptosis (O'Rourke et al. 2005). Accumulating evidence indicate oxidative stress involves in the pathophysiologic hippocampus injury and plays a vital role in the CUMS-induced pathogenesis (Ahmad et al. 2010; Sakr et al. 2015; Chen et al. 2016; Jindal et al. 2013; Bhatt et al. 2014). The inflammatory stimuli are likely to be in response to apoptosis (Zonis et al. 2013; Zunszain et al. 2012). Fortunately, in current study, we found that PB inhibited the TNF- $\alpha$ and IL-1 $\beta$ expression, but increase the expression of TGF- $\beta$ and IL-10, indicating that the PB might ameliorate 


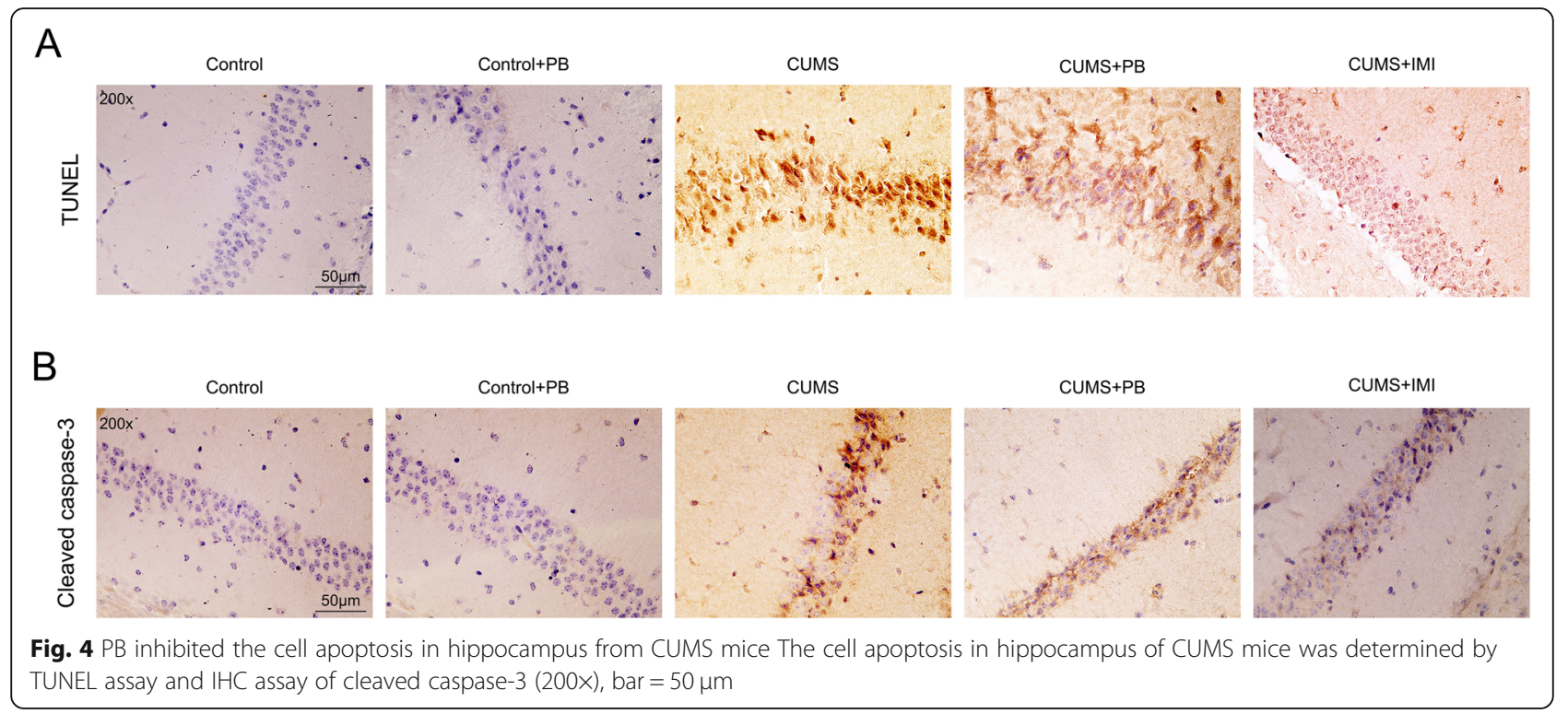

neuroinflammation in CUMS-induced mice. Moreover, PB decreased the concentrations of ROS and MDA, but increased the SOD activity, suggesting that its protective function to alleviate oxidative stress. Thus, combined all these findings, we concluded that PB alleviate depressive-like behaviors via inhibiting oxidative stress and neuroinflammation, resulting in reducing cell apoptosis in CUMS-induced mice. Nevertheless, how PB influences the neuroinflammation and oxidative stress remains unclear.
There are many evidences highlight that $\mathrm{PB}$ serves as a target for Nrf2/HO-1 Axis, reduces autonomic dysfunction and susceptibility to atrial fibrillation via deactivating NF- $\mathrm{KB} / \mathrm{TNF}-\alpha$ pathway, inhibits the proliferation and migration but promotes the apoptosis through inhibiting N-cadherin and GABAB receptor (Ye et al. 2019; Gao et al. 2019; Habtemariam 2019). Interestingly, we confirmed that PB activated Nrf2/HO-1 signal pathway but inhibited the phosphorylation of NF-kB, which was partly consistent with previous studies. Nrf2-ARE

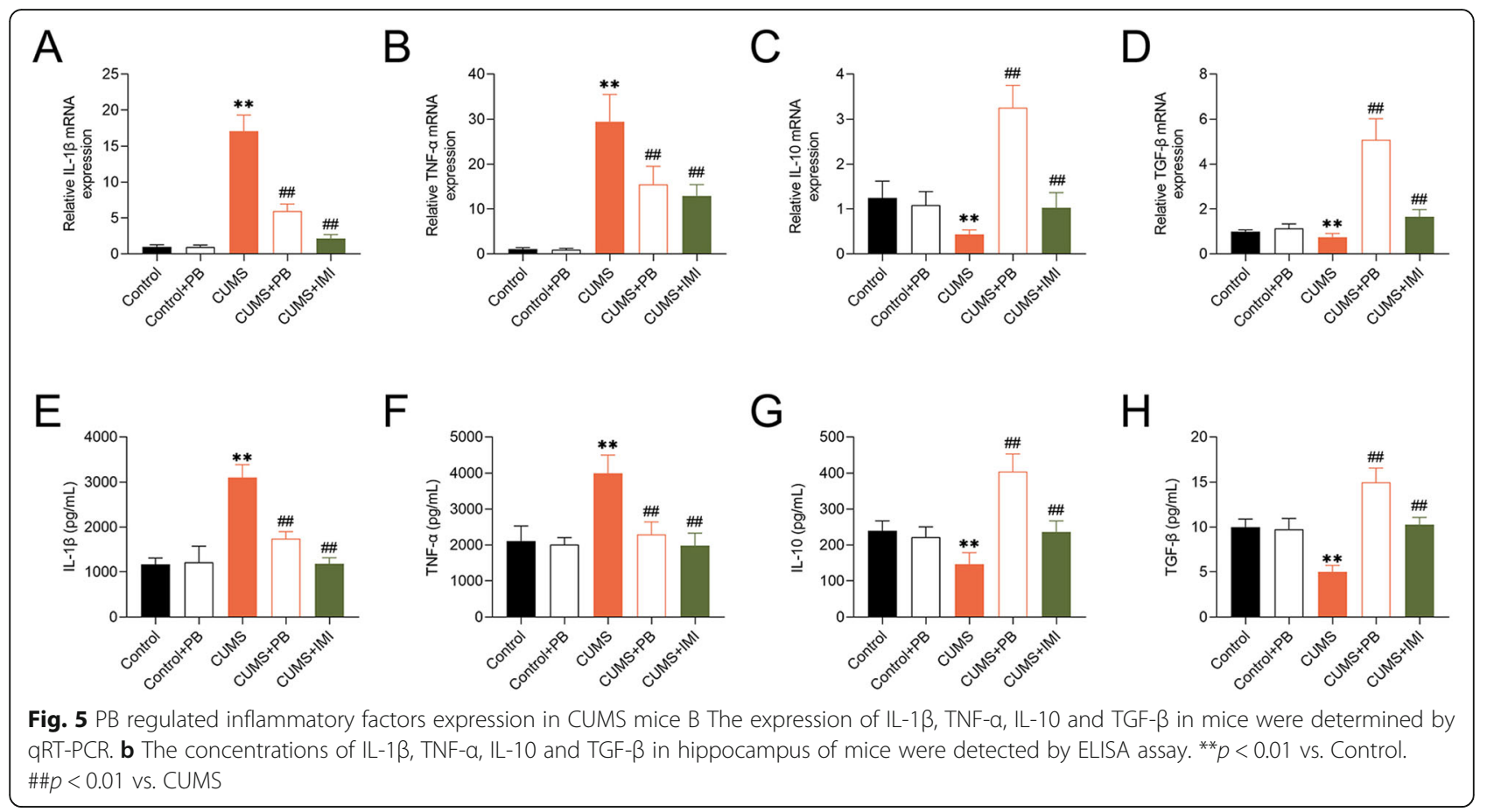




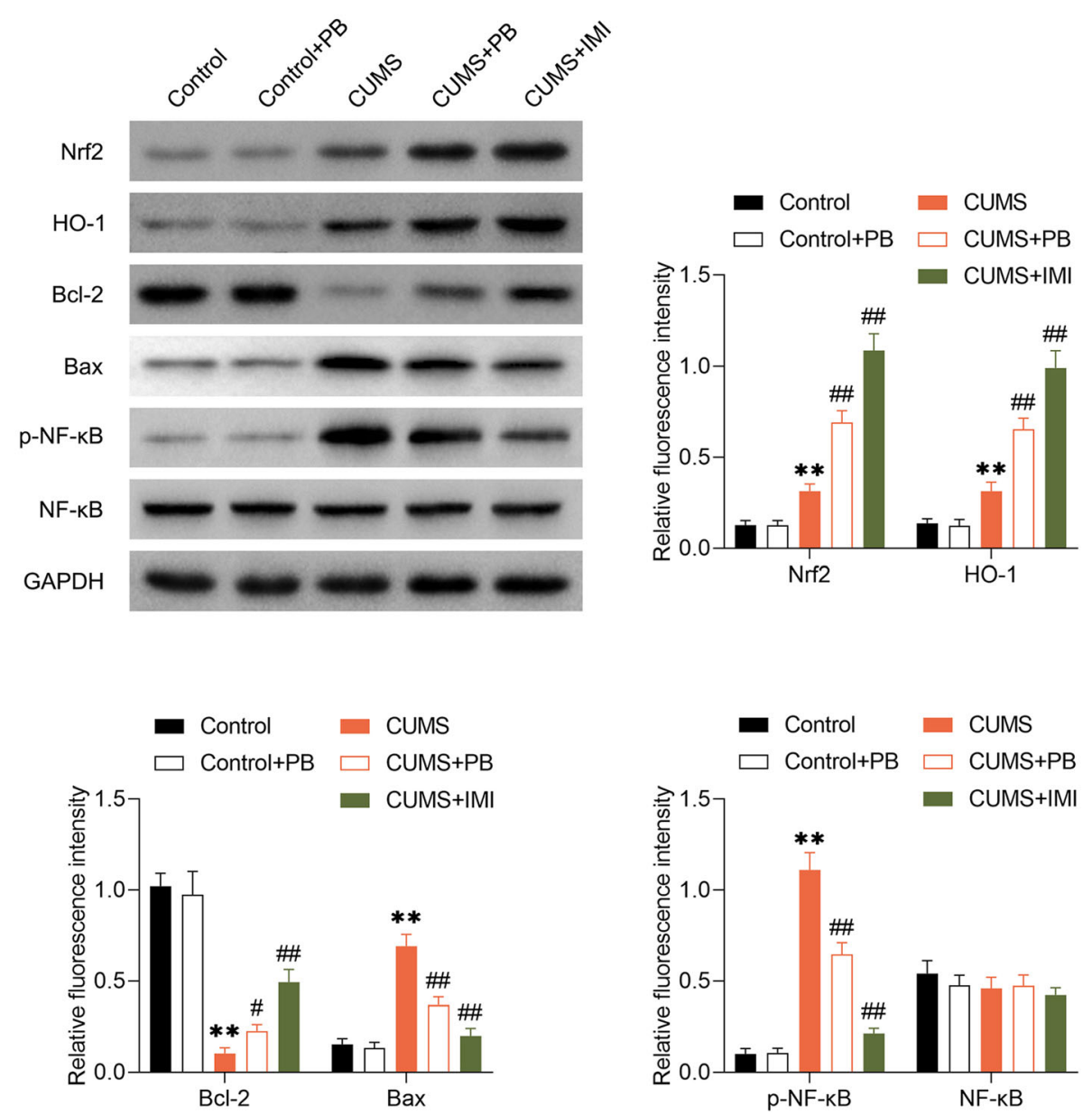

Fig. 6 PB activated Nrf2/HO-1 signal pathway but inhibited the phosphorylation of NF-kB The changes of expression of Nrf2, HO-1, Bcl-2, Bax, pNF-kB, NF-kB were assessed by western blot. ${ }^{* *} p<0.01$ vs. Control. \#p $<0.05, \# \# p<0.01$ vs. CUMS

pathway is proved to reduce oxidative stress and neuroinflammation, and play a protective role in neurodegenerative diseases (Buendia et al. 2016). Moreover, upregulation of NF-kB perpetuates oxidative stress and contributes to neuroinflammation (Tobon-Velasco et al.
2014). However, how PB activates Nrf2/HO-1 signal pathway but inhibits the phosphorylation of NF-kB is still known, whether it could regulate some miRNAs or expression of proteins and then participate in $\mathrm{Nrf} 2 / \mathrm{HO}$ 1 or NF-kB signal pathways? Hence, it still should pay

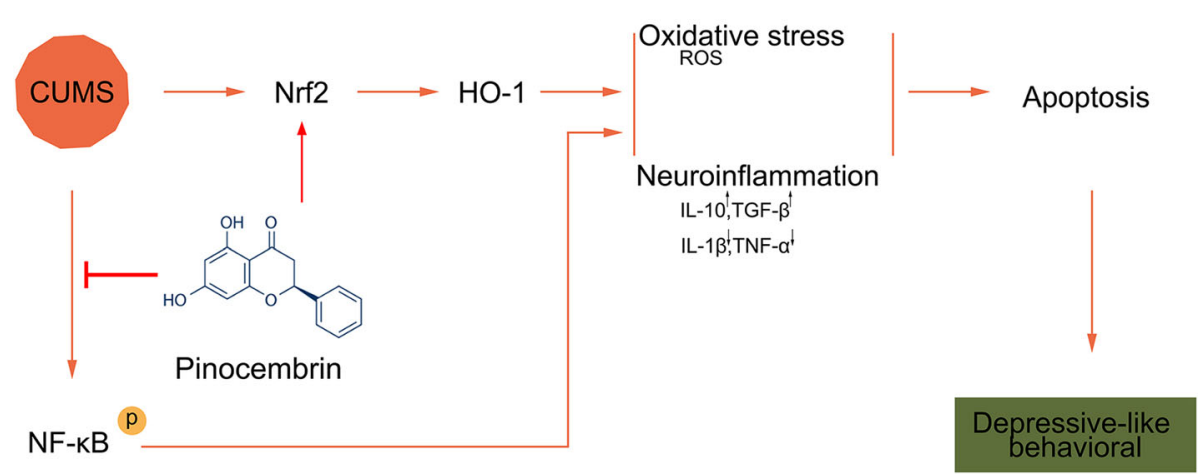

Fig. 7 This cartoon depicted PB activated Nrf2/HO-1 signal pathway but inhibited the phosphorylation of NF-kB, and then ameliorated neuroinflammation and oxidative stress, further inhibited cell apoptosis and mitigated CUMS-induced depressive-like behaviors 
more efforts for us to investigate the potential mechanism of PB in depression in clinic and animal models. Moreover, given that serum biomarkers relate to the degree of inflammation at brain site, we suspect that PB treatment might be associated with changes in peripheral inflammatory serum, which may provide a novel sight for explain the mechanism of $\mathrm{PB}$ in depression (Lattanzi et al. 2020; Di Napoli et al. 2018; Lattanzi et al. 2019).

\section{Conclusion}

In conclusion, the present study demonstrated that $\mathrm{PB}$ reduces CUMS-induced hippocampal neuroinflammation and oxidative stress, thus inhibits cell apoptosis via activating Nrf2/HO-1 signal pathway but inhibited the phosphorylation of NF-kB. Our study proved an important role of $\mathrm{PB}$ against CUMS-induced neuroinflammation and apoptosis via Nrf2/HO-1 and NF-kB signaling pathway and provide a basis for investigating $\mathrm{PB}$ as a therapeutic strategy for the pathogenesis of depression.

\section{Abbreviation}

PB: Pinocembrin; CUMS: Chronic unpredictable mild stress; SPT: Sucrose preference test; OFT: Open field test; FST: Forced swim test; TST: Tail suspension test; ROS: Oxygen species; MDA: Malondialdehyde; SOD: Superoxide dismutase; IL: Inflammatory factor including interleukin; TNF: Tumor necrosis factor; TGF: Transforming growth factor

\section{Acknowledgements}

Not applicable.

\section{Authors' contributions}

WW and LLZ conceived and designed the experiments, LJX and JLT analyzed and interpreted the results of the experiments, XHG performed the experiments. The author(s) read and approved the final manuscript.

\section{Funding}

This work was supported by the National Natural Science Funds of China (Grant No.81760256 and 81960256) and Natural Science Foundation of Jiangxi, China (Grant No. 20192BAB205038) to Dr. Wei Wang.

\section{Availability of data and materials}

All data generated or analyzed during this study are included in this published article.

\section{Ethics approval and consent to participate}

This study was obeyed the Guide for the Care and Use of Laboratory Animals and the protocol was approved by the Ethics Committee of The Second Affiliated Hospital of Nanchang University.

\section{Consent for publication}

Not applicable.

\section{Competing interests}

The authors state that there are no conflicts of interest to disclose.

\section{Author details}

${ }^{1}$ Department of Neurology, The Second Affiliated Hospital of Nanchang University, No.1 Minde Road, Nanchang City, Jiangxi Province, China. ${ }^{2}$ Department of Pharmacy, Jiangxi Maternal and Child Health Hospital, Nanchang City 330006, Jiangxi Province, China.
Received: 10 March 2020 Accepted: 7 May 2020

Published online: 27 May 2020

\section{References}

Ahmad A, Rasheed N, Banu N, Palit G. Alterations in monoamine levels and oxidative systems in frontal cortex, striatum, and hippocampus of the rat brain during chronic unpredictable stress. Stress. 2010;13(4):355-64.

Al-Hakeim HK, Al-Rammahi DA, Al-Dujaili AH. IL-6, IL-18, sIL-2R, and TNFalpha proinflammatory markers in depression and schizophrenia patients who are free of overt inflammation. J Affect Disord. 2015;182:106-14.

Belzung C, Griebel G. Measuring normal and pathological anxiety-like behaviour in mice: a review. Behav Brain Res. 2001;125(1-2):141-9.

Bhatt S, Radhakrishnan M, Jindal A, Devadoss T, Dhar AK. Neuropharmacological evaluation of a novel 5-HT3 receptor antagonist $(6 \mathrm{~g})$ on chronic unpredictable mild stress-induced changes in behavioural and brain oxidative stress parameters in mice. Indian J Pharm. 2014;46(2):191-6 PMID: 3987189.

Buendia I, Michalska P, Navarro E, Gameiro I, Egea J, Leon R. Nrf2-ARE pathway: an emerging target against oxidative stress and neuroinflammation in neurodegenerative diseases. Pharmacol Ther. 2016:157:84-104.

Butter S, Shevlin M, Murphy J. Negative self-evaluation and the genesis of internal threat: beyond a continuum of suicidal thought and behaviour. Psychol Med. 2019;49(15):2591-9.

Chen H, Zhou L, Wu X, Li R, Wen J, Sha J, et al. The PI3K/AKT pathway in the pathogenesis of prostate cancer. Front Biosci (Landmark Ed). 2016;21:108491.

Coleman JRI, Gaspar HA, Bryois J, Bipolar Disorder Working Group of the Psychiatric Genomics C, Major Depressive Disorder Working Group of the Psychiatric Genomics C, Breen G. The Genetics of the Mood Disorder Spectrum: Genome-wide Association Analyses of More Than 185,000 Cases and 439,000 Controls. Biol Psychiatry. 2019. https://doi.org/10.1016/j.biopsych. 2019.10.015.

Cryan JF, Mombereau C, Vassout A. The tail suspension test as a model for assessing antidepressant activity: review of pharmacological and genetic studies in mice. Neurosci Biobehav Rev. 2005:29(4-5):571-625.

Di Napoli M, Slevin M, Popa-Wagner A, Singh P, Lattanzi S, Divani AA. Monomeric C-Reactive Protein and Cerebral Hemorrhage: From Bench to Bedside. Front Immunol. 2018:9:1921 PMID:6141664.

Eyre HA, Air T, Pradhan A, Johnston J, Lavretsky H, Stuart MJ, et al. A metaanalysis of chemokines in major depression. Prog Neuro-Psychopharmacol Biol Psychiatry. 2016;68:1-8 PMID:5536843.

Gao J, Lin S, Gao Y, Zou X, Zhu J, Chen M, et al. Pinocembrin inhibits the proliferation and migration and promotes the apoptosis of ovarian cancer cells through down-regulating the mRNA levels of N-cadherin and GABAB receptor. Biomed Pharmacother. 2019;120:109505.

Habtemariam S. The Nrf2/HO-1 Axis as Targets for Flavanones: Neuroprotection by Pinocembrin, Naringenin, and Eriodictyol. Oxidative Med Cell Longev. 2019:2019:4724920 PMID:6878820.

Hannestad J, DellaGioia N, Bloch M. The effect of antidepressant medication treatment on serum levels of inflammatory cytokines: a meta-analysis. Neuropsychopharmacology. 2011;36(12):2452-9 PMID:3194072.

Hemmati AA, Alboghobeish S, Ahangarpour A. Effects of cinnamic acid on memory deficits and brain oxidative stress in streptozotocin-induced diabetic mice. Korean J Physiol Pharmacol. 2018;22(3):257-67 PMID:5928339.

Jiang P, Guo Y, Dang R, Yang M, Liao D, Li H, et al. Salvianolic acid B protects against lipopolysaccharide-induced behavioral deficits and neuroinflammatory response: involvement of autophagy and NLRP3 inflammasome.J Neuroinflammation. 2017a:14(1):239 PMID:5719935.

Jiang X, Liu J, Lin Q, Mao K, Tian F, Jing C, et al. Proanthocyanidin prevents lipopolysaccharide-induced depressive-like behavior in mice via neuroinflammatory pathway. Brain Res Bull. 2017b;135:40-6.

Jindal A, Mahesh R, Bhatt S. Etazolate, a phosphodiesterase 4 inhibitor reverses chronic unpredictable mild stress-induced depression-like behavior and brain oxidative damage. Pharmacol Biochem Behav. 2013;105:63-70.

Kessler RC, Bromet EJ. The epidemiology of depression across cultures. Annu Rev Public Health. 2013;34:119-38 PMID:4100461.

Kubera M, Obuchowicz E, Goehler L, Brzeszcz J, Maes M. In animal models, psychosocial stress-induced (neuro) inflammation, apoptosis and reduced neurogenesis are associated to the onset of depression. Prog NeuroPsychopharmacol Biol Psychiatry. 2011;35(3):744-59. 
Kwon DH, Kim BS, Chang H, Kim Yl, Jo SA, Leem YH. Exercise ameliorates cognition impairment due to restraint stress-induced oxidative insult and reduced BDNF level. Biochem Biophys Res Commun. 2013;434(2):245-51.

Lan X, Han X, Li Q, Li Q, Gao Y, Cheng T, et al. Pinocembrin protects hemorrhagic brain primarily by inhibiting toll-like receptor 4 and reducing M1 phenotype microglia. Brain Behav Immun. 2017:61:326-39 PMID:5453178.

Lan X, Wang W, Li Q, Wang J. The Natural Flavonoid Pinocembrin: Molecular Targets and Potential Therapeutic Applications. Mol Neurobiol. 2016;53(3): 1794-801 PMID:4561606.

Lattanzi S, Brigo F, Trinka E, Cagnetti C, Di Napoli M, Silvestrini M. Neutrophil-tolymphocyte ratio in acute cerebral hemorrhage: a system review. Transl Stroke Res. 2019;10(2):137-45.

Lattanzi S, Di Napoli M, Ricci S, Divani AA. Matrix Metalloproteinases in Acute Intracerebral Hemorrhage. Neurotherapeutics. 2020. https://doi.org/10.1007/ s13311-020-00839-0.

Lee WY, Jang SW, Lee JS, Kim YH, Kim HG, Han JM, et al. Uwhangchungsimwon a traditional herbal medicine, protects brain against oxidative injury via modulation of hypothalamus-pituitary-adrenal (HPA) response in a chronic restraint mice model. J Ethnopharmacol. 2014;151(1):461-9.

Liu MY, Yin CY, Zhu LJ, Zhu XH, Xu C, Luo CX, et al. Sucrose preference test for measurement of stress-induced anhedonia in mice. Nat Protoc. 2018;13(7): 1686-98.

Madrigal JL, Garcia-Bueno B, Caso JR, Perez-Nievas BG, Leza JC. Stress-induced oxidative changes in brain. CNS Neurol Disord Drug Targets. 2006;5(5):561-8.

McKernan DP, Dinan TG, Cryan JF. "Killing the blues": a role for cellular suicide (apoptosis) in depression and the antidepressant response? Prog Neurobiol. 2009;88(4):246-63.

Newman DJ, Cragg GM, Snader KM. Natural products as sources of new drugs over the period 1981-2002. J Nat Prod. 2003;66(7):1022-37.

O'Rourke B, Cortassa S, Aon MA. Mitochondrial ion channels: gatekeepers of life and death. Physiology (Bethesda). 2005;20:303-15 PMID:2739045.

Peng $\mathrm{H}$. Wang $\mathrm{H}-\mathrm{b}$, Wang L, Zhou B, Li X-y, tan J.Gsk3 $\beta$ aggravates the depression symptoms in chronic stress mouse model. J Integr Neurosci. 2018;17(2):217-26.

Rasul A, Millimouno FM, Ali Eltayb W, Ali M, Li J, Li X. Pinocembrin: a novel natural compound with versatile pharmacological and biological activities. Biomed Res Int. 2013;2013:379850 PMID:3747598.

Reddy KK, Grossman L, Rogers GS. Common complementary and alternative therapies with potential use in dermatologic surgery: risks and benefits. J Am Acad Dermatol. 2013;68(4):e127-35.

Riddle M, Potter GG, McQuoid DR, Steffens DC, Beyer JL, Taylor WD. Longitudinal Cognitive Outcomes of Clinical Phenotypes of Late-Life Depression. Am J Geriatr Psychiatry. 2017;25(10):1123-34 PMID:5600662.

Saad MA, Abdel Salam RM, Kenawy SA, Attia AS. Pinocembrin attenuates hippocampal inflammation, oxidative perturbations and apoptosis in a rat model of global cerebral ischemia reperfusion. Pharmacol Rep. 2015;67(1): $115-22$.

Sakr HF, Abbas AM, Elsamanoudy AZ, Ghoneim FM. Effect of fluoxetine and resveratrol on testicular functions and oxidative stress in a rat model of chronic mild stress-induced depression. J Physiol Pharmacol. 2015;66(4):515-27.

Salim S. Oxidative Stress and the Central Nervous System. J Pharmacol Exp Ther 2017;360(1):201-5 PMID:5193071.

Stockmeier CA, Mahajan GJ, Konick LC, Overholser JC, Jurjus GJ, Meltzer HY, et al. Cellular changes in the postmortem hippocampus in major depression. Biol Psychiatry. 2004;56(9):640-50 PMID:2929806.

Tell G, Gustincich S. Redox state, oxidative stress, and molecular mechanisms of protective and toxic effects of bilirubin on cells. Curr Pharm Des. 2009;15(25): 2908-14.

Tobon-Velasco JC, Cuevas E, Torres-Ramos MA. Receptor for AGEs (RAGE) as mediator of NF-kB pathway activation in neuroinflammation and oxidative stress. CNS Neurol Disord Drug Targets. 2014;13(9):1615-26.

Wu X, Tang B, Liao X, Su Z, Lee SM, Cai Y, et al. Suppressive effects of the supercritical-carbon dioxide fluid extract of Chrysanthemum indicum on chronic unpredictable mild stress-induced depressive-like behavior in mice. Food Funct. 2019;10(2):1212-24

Yamawaki Y, Yoshioka N, Nozaki K, Ito H, Oda K, Harada K, et al. Sodium butyrate abolishes lipopolysaccharide-induced depression-like behaviors and hippocampal microglial activation in mice. Brain Res. 2018;1680:13-38.

Yankelevitch-Yahav R, Franko M, Huly A, Doron R. The forced swim test as a model of depressive-like behavior. J Vis Exp. 2015;(97). https://doi.org/10. 3791/52587 PMID:4401172.
Ye T, Zhang C, Wu G, Wan W, Liang J, Liu X, et al. Pinocembrin attenuates autonomic dysfunction and atrial fibrillation susceptibility via inhibition of the NF-kappaB/TNF-alpha pathway in a rat model of myocardial infarction. Int Immunopharmacol. 2019;77:105926.

Zonis S, Ljubimov VA, Mahgerefteh M, Pechnick RN, Wawrowsky K, Chesnokova V. p21Cip restrains hippocampal neurogenesis and protects neuronal progenitors from apoptosis during acute systemic inflammation. Hippocampus. 2013;23(12):1383-94 PMID:4170945.

Zunszain PA, Anacker C, Cattaneo A, Choudhury S, Musaelyan K, Myint AM, et al. Interleukin-1 beta: a new regulator of the kynurenine pathway affecting human hippocampal neurogenesis. Neuropsychopharmacology. 2012;37(4): 939-49 PMID:3280640.

\section{Publisher's Note}

Springer Nature remains neutral with regard to jurisdictional claims in published maps and institutional affiliations.
Ready to submit your research? Choose BMC and benefit from:

- fast, convenient online submission

- thorough peer review by experienced researchers in your field

- rapid publication on acceptance

- support for research data, including large and complex data types

- gold Open Access which fosters wider collaboration and increased citations

- maximum visibility for your research: over $100 \mathrm{M}$ website views per year

At BMC, research is always in progress.

Learn more biomedcentral.com/submissions 